\title{
Estadificación locorregional por resonancia magnética del cáncer de recto: influencia de la imagen potenciada en difusión en la precisión diagnóstica
}

\author{
Locoregional staging using magnetic resonance imaging in rectal cancer: influence of \\ diffusion-weighted imaging in the diagnostic accuracy
}

\author{
Roberto Fornell-Pérez ${ }^{*}$, Patricia Alemán-Flores², Gabriela Porcel-de Peralta², Jano Rubio-García², \\ María C. González-Domínguez ${ }^{2}$, Joel Aranda-Sánchez ${ }^{2}$, Valentina Vivas-Escalona ${ }^{2}$ y Juan F. Loro-Ferrer ${ }^{1}$ \\ ${ }^{1}$ Departamento de Ciencias Clínicas, Facultad de Medicina, Universidad de Las Palmas de Gran Canaria; ${ }^{2}$ Servicio de Radiodiagnóstico, Complejo \\ Hospitalario Universitario Insular Materno-Infantil. Las Palmas de Gran Canaria, España
}

\begin{abstract}
Resumen
Objetivo: Evaluar si la eficacia en la estadificación locorregional por resonancia magnética (RM) del cáncer de recto (primaria o posneoadyuvancia) mejora al añadir imágenes potenciadas en difusión, según la experiencia previa del radiólogo. Método: Estudio retrospectivo sobre 100 RM de 1.5 T (2011-2016) de pacientes con cáncer rectal (estándar de referencia: estadiaje histológico de pieza quirúrgica). Diez radiólogos (tres con experiencia en cáncer rectal, tres inexpertos y cuatro residentes) evaluaron individualmente cada caso dos veces: primero, solo secuencias T2 de alta resolución; segundo, valoración conjunta con difusión. Se analizó la diferenciación entre estadios precoces (0-I) y avanzados (II-IV), y se calcularon la precisión, la sensibilidad y la especificidad, y los valores predictivos. Resultados: Al agregar la difusión, los radiólogos experimentados presentaron peores resultados, sobre todo en estadiaje primario (precisión: 0.769 a 0.701). Los inexpertos mostraron mejoría posneoadyuvancia (precisión: 0.574 a 0.642; especificidad: 19.1 a 29.8\%), sin otros cambios destacables. Los residentes manifestaron peores resultados en estadiaje primario (precisión: 0.670 a 0.633; especificidad: 45.8-39.6\%), pero mejoría posneoadyuvancia (sensibilidad: 80.6 a $87 \%$ ). Las diferencias entre ambas revisiones no fueron estadísticamente significativas. Conclusiones: No se encontraron diferencias significativas en la distinción entre tumores rectales precoces y avanzados al añadir secuencias de difusión al uso de secuencias T2 de alta resolución.
\end{abstract}

Palabras clave: Invasividad tumoral. Neoplasia rectal. Quimioterapia neoadyuvante. Resonancia magnética. Secuencias potenciadas en difusión.

\begin{abstract}
Objective: To assess whether the accuracy in locoregional staging using magnetic resonance imaging (MRI) in rectal cancer (primary or post-chemoradiotherapy) improves by adding diffusion-weighted imaging, according to the radiologist's degree of experience. Method: Retrospective study on $100 \mathrm{MRI}$ records (1.5 T, 2011-2016) from patients with rectal cancer (reference standard: histology of surgical specimens). Ten radiologists (three experienced in rectal cancer, three specialized in other areas and four residents) individually reviewed each case twice: first, evaluating just high-resolution T2-weighted sequences; second,
\end{abstract}

\footnotetext{
Correspondencia:

*Roberto Fornell-Pérez

Avda. Marítima, $\mathrm{s} / \mathrm{n}$

Fecha de recepción: 26-11-2019

C.P. 35016, Las Palmas de Gran Canaria, España Fecha de aceptación: 20-03-2020

E-mail: feanim2000@yahoo.es

DOI: $10.24875 / C I R U .20001719$

Cir Cir. 2020;88(4):420-427

Contents available at PubMed

www.cirugiaycirujanos.com

0009-7411/@ 2020 Academia Mexicana de Cirugía. Publicado por Permanyer. Este es un artículo open access bajo la licencia CC BY-NC-ND (http://creativecommons.org/licenses/by-nc-nd/4.0/).
} 
evaluation of diffusion-weighted plus high-resolution ones. The analysis focused on the differentiation between early (0-I) and advanced (II-IV) stages. Accuracy, sensitivity/specificity and predictive values were calculated. Results: Experienced radiologists showed some worsening by adding diffusion-weighted imaging, mainly at primary staging (accuracy: 0.769 to 0.701 ). Inexperienced radiologists presented a post-chemoradiotherapy improvement (accuracy: 0.574 to 0.642 ; specificity of 19.1 to $29.8 \%$ ), although with no other remarkable changes. Residents demonstrated a worsening at primary staging by adding diffusion (accuracy: 0.670 to 0.633 ; specificity: 45.8 to $39.6 \%$ ), but post-chemoradiotherapy improvement (sensitivity: 80.6 to $87 \%$ ). The differences between both reviews were not statistically significant. Conclusions: No significant differences were found in the distinction between early and advanced rectal tumors secondary to adding diffusion-weighted imaging to high-resolution T2-weighted sequences.

Key words: Neoplasm invasiveness. Rectal neoplasms. Neoadjuvant chemotherapy. Magnetic resonance imaging. Diffusion-weighted imaging.

\section{Introducción}

El abordaje del cáncer rectal ha sufrido marcados cambios en las últimas décadas. Los avances en técnicas quirúrgicas menos agresivas y en el tratamiento neoadyuvante con quimiorradioterapia (QRT) han logrado un importante aumento de la supervivencia y una disminución de la morbilidad y de la recidiva local ${ }^{1-3}$. Para la correcta toma de decisiones resulta esencial un estadiaje previo por imagen lo más preciso posible, donde la resonancia magnética (RM) ha demostrado su valor ${ }^{4-6}$.

Hoy en día sigue habiendo puntos controvertidos acerca de la RM aplicada al cáncer rectal, como por ejemplo la utilidad de las secuencias potenciadas en difusión $(\mathrm{DWI})^{7,8}$. Si bien existe acuerdo en que las secuencias T2 de alta resolución (HRT2w) son esenciales para su valoración, se ha planteado el valor potencial de la DWI para la evaluación post-QRT o la detección de respuesta completa ${ }^{9-13}$. Persiste cierto debate sobre su uso en el estadiaje primario o ganglionar ${ }^{14-16}$; un reciente artículo de consenso no recomienda la DWI en dichos casos, pero metaanálisis previos destacan la escasez y la heterogeneidad de la evidencia disponible 5,11,17. $^{\text {. }}$.

Existen muy pocos estudios sobre la influencia de la DWI en el estadiaje locorregional (TN), a pesar de su uso habitual en la práctica diaria y su relevancia clínica en la toma de decisiones ${ }^{18}$. Igualmente, hay pocos datos sobre su rendimiento en radiólogos menos experimentados, en quienes la DWI presentaría potencialmente mayor interés al facilitar la visualización y delimitación del tumor y la detección de ganglios perirrectales ${ }^{12,15,19,20}$.

El objetivo de nuestro estudio fue evaluar si añadir secuencias DWI mejora la eficacia diagnóstica de las secuencias HRT2w para diferenciar mediante RM estadios precoces (0-I) de estadios avanzados (II-IV) en el cáncer rectal, así como valorar si existen diferencias entre radiólogos expertos en RM rectal y otros menos expertos.

\section{Método}

Estudio retrospectivo transversal basado en RM de estadificación de cáncer rectal realizadas en nuestro centro. El estudio fue aprobado por el Comité Hospitalario de Ética en Investigación; dada su naturaleza retrospectiva, no se solicitó consentimiento informado.

\section{Pacientes}

Las RM se realizaron entre enero de 2011 y julio de 2016, y fueron incluidas consecutivamente hasta alcanzar una muestra final de 100 (Fig. 1). El estudio abarcó pacientes tanto con cirugía tras el estadiaje primario como con tratamiento neoadyuvante previo a la cirugía (post-QRT). Los criterios de inclusión fueron: a) cáncer de recto confirmado mediante colonoscopia y biopsia; b) RM rectal con una técnica correcta de acuerdo con el protocolo establecido, con todas las secuencias disponibles para revisión y sin artefactos significativos; c) RM de seguimiento post-QRT cuando se realizó tratamiento neoadyuvante; y d) escisión mesorrectal total o resección abdominoperineal tras la RM con pieza quirúrgica completa. En la estadificación primaria, el período máximo admitido hasta la cirugía fue de 9 semanas (media: 34.7 días). En los casos post-QRT, se incluyeron aquellos con cirugía entre 6 y 10 semanas después del final del tratamiento (media: 64.1 días). La RM de seguimiento post-QRT fue la considerada en el estudio cuando se realizó tratamiento neoadyuvante; se excluyeron los casos con un período de 5 semanas o menos entre el final de la QRT y la RM de seguimiento (media: 40.7 días).

La necesidad de QRT se valoró por el Comité Hospitalario de Tumores Colorrectales. En general, no se consideraron indicación de QRT los estadios IIA 


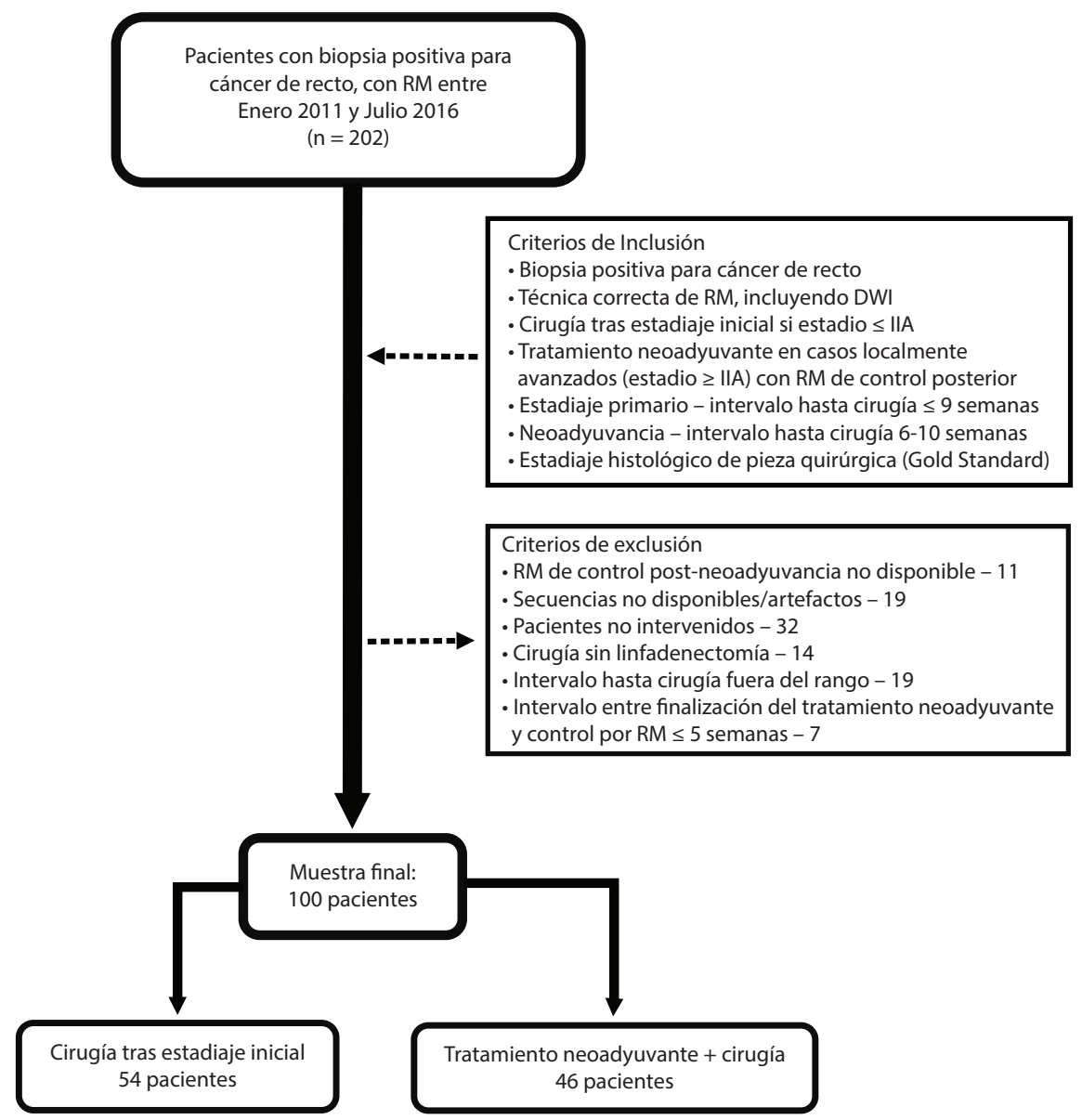

Figura 1. Flujo de trabajo del estudio.

con infiltración superficial por imagen, sin factores de mal pronóstico. Los casos con tumores localizados en el tercio superior del recto o en la unión rectosigmoidea se evaluaron individualmente ${ }^{3}$. La pauta de neoadyuvancia consistió en $825 \mathrm{mg} / \mathrm{m}^{2}$ cada 12 horas de capecitabina por vía oral, concomitante con un ciclo largo de radioterapia ( 50.4 Gy en 25 sesiones) ${ }^{21}$.

Para el análisis histopatológico, las muestras quirúrgicas se fijaron en formalina durante 24 horas. Se tomaron cortes representativos del área del tumor y de las posibles zonas de infiltración; todos los ganglios detectados se aislaron de la grasa perirrectal y se incluyeron por entero. Dos patólogos (con 7 y 10 años de experiencia en tumores colorrectales) establecieron el estadio anatomopatológico, que fue el estándar de referencia del estudio.

\section{Protocolo de RM}

Los estudios se realizaron en un equipo de RM de 1.5 T (Magnetom Avanto; Siemens) con bobina de superficie
Tabla 1. Parámetros técnicos del protocolo de resonancia magnética utilizado en el estudio

\begin{tabular}{lccc}
\hline & \multicolumn{1}{c}{ T2w } & HRT2w & DWI \\
\hline Plano & $\begin{array}{c}\text { Axial, coronal, } \\
\text { sagital }\end{array}$ & $\begin{array}{c}\text { Axial, coronal, } \\
\text { sagital }\end{array}$ & Axial \\
Referencia & Pelvis & $\begin{array}{c}\text { Eje del tumor } \\
\text { TR/TE }\end{array}$ & Pelvis \\
Grosor de corte & $6700 / 95 \mathrm{~ms}$ & $4000 / 95 \mathrm{~ms}$ & $5000 / 70 \mathrm{~ms}$ \\
Matriz & $256 \times 230$ & $256 \times 230$ & $192 \times 115$ \\
FOV & 340 & 200 & 300 \\
Duración & $150 \mathrm{~s}$ & $135 \mathrm{~s}$ & $200 \mathrm{~s}$ \\
\hline
\end{tabular}

DWI: secuencias potenciadas en difusión; FOV: field of view; HRT2w: secuencia T2 de alta resolución;TR/TE: tiempo de repetición/tiempo de eco; T2w: secuencia T2.

de 16 canales. A todos los pacientes se les administró previamente $50 \mathrm{ml}$ de gel por vía rectal y $20 \mathrm{mg}$ de bromuro de butilescopolamina por vía intramuscular. El protocolo de RM utilizado se muestra en la tabla 1. 


\section{Evaluación de las imágenes}

Las imágenes se valoraron en monitores de alta resolución con Picture Archiving and Communication System (PACS; General Electric). Cada caso fue evaluado por 10 radiólogos con diferentes grados de experiencia en la estadificación del cáncer de recto por RM: tres (ER) con experiencia previa de 6, 5 y 3 años (aproximadamente 40 casos/año); otros tres (NER) con 7, 4 y 2 años de experiencia en RM en áreas diferentes a la gastrointestinal; y cuatro residentes de radiología (RR) en sus últimos años de especialización, con conocimientos generales sobre RM abdominal. Al comienzo del estudio, los NER y los RR recibieron un entrenamiento básico (2 horas) por parte de un radiólogo experimentado, con revisión y discusión de varios ejemplos del mismo centro.

Todos ellos revisaron los casos de forma individual y ciega, salvo por el antecedente de biopsia positiva para neoplasia rectal. La revisión de cada caso se llevó a cabo en dos fases: la primera se basó en el análisis únicamente con secuencias HRT2w, y tras un período de lavado mínimo de un mes y aleatorización, la segunda fase consistió en una nueva valoración de cada caso, esta vez con secuencias tanto HRT2w como DWI.

La valoración del tumor principal con HRT2w se basó en la presencia o la persistencia de focos de engrosamiento o masas sólidas de intensidad intermedia en la pared rectal, considerando como signo de infiltración un margen borroso con lobulaciones 0 extensión hacia la grasa adyacente. La aparición de una marcada hipointensidad en la RM de seguimiento post-QRT se consideró indicativa de fibrosis. Con DWI, el criterio de sospecha de malignidad fue la presencia o la persistencia de una alta intensidad de señal en valores altos de $b$ con hipointensidad en ADC. Para la determinación de afectación extramural se correlacionaron los hallazgos con su localización en HRT2w.

En la valoración de los ganglios regionales, los criterios de malignidad en HRT2w fueron morfológicos: presencia de heterogeneidad de señal intranodal, márgenes externos irregulares o forma redondeada ${ }^{22}$. Cada ganglio se valoró mediante una escala de confianza de 1 a 3 ( 1 = maligno; 2 = dudoso; 3 = benigno), con un recuento del número de ganglios por grado en cada paciente; la presencia de dos o más criterios se consideró sugestiva de malignidad, mientras que uno solo se clasificó como dudoso. En DWI se consideró como criterio de sospecha la presencia de hiperintensidad del ganglio en valores altos de $b$ en comparación con la señal de fondo suprimida de la grasa mesorrectal, asociada a hipointensidad difusa o focal en ADC. Para la evaluación conjunta con HRT2w y DWI se aportaba un grado de sospecha en la escala basado en HRT2w y, en caso de existir sospecha en DWI, se bajó un grado la puntuación (por ejemplo, HRT2w dudoso + sospecha en DWI = maligno). El tamaño del ganglio no se incluyó, ya que durante el diseño y la recogida de datos no se habían publicado límites claros ni criterios mixtos ${ }^{9,23}$. Los ganglios laterales no se evaluaron, pues la técnica quirúrgica no siempre incluyó una linfadenectomía sistemática.

Basándose en estos datos, se determinó el estadio locorregional de ambas fases paciente a paciente, de acuerdo con las directrices del American Joint Committee on Cancer (AJCC) ${ }^{24}$. Para ello solo se consideraron los ganglios descritos como grado 1 (malignos) de la escala.

\section{Análisis estadístico}

Se realizó con los paquetes estadísticos IBM SPSS Statistics 24.0 (IBM Corp), Epidat 4.1 (SERGAS, Xunta de Galicia) y MedCalc 12.2.1 (Ostend). Los resultados se agruparon en las diferentes fases según el grado de experiencia, calculando los valores medios del grupo.

Los datos histológicos se dicotomizaron en estadios $0-I$ y estadios II-IV, con el objetivo de valorar el rendimiento en la detección de tumores localmente avanzados. La elección del punto de corte se hizo teniendo en cuenta los cambios terapéuticos que pueden derivar de este: en el estadiaje primario, la necesidad de QRT previa a la cirugía; en el control post-QRT, la variación de factores pronósticos y la posibilidad de técnicas quirúrgicas menos agresivas en caso de reducción de la extensión tumoral ${ }^{3}$.

La precisión diagnóstica se valoró mediante el cálculo del área bajo la curva (AUC) ROC (Receiver Operating Characteristic) para cada revisión y observador. Además, se determinaron la sensibilidad, la especificidad, el valor predictivo positivo (VPP), el valor predictivo negativo (VPN), las razón de verosimilitud positiva (RVP) y la razón de verosimilitud negativa (RVN). Su significación estadística se evaluó mediante la prueba exacta de Fisher $(p<0.05)$, y la significación de las diferencias entre valores con el método de DeLong (en curvas ROC) y el test de McNemar (resto de los parámetros). También se calcularon las tasas de 
Tabla 2. Datos demográficos y sobre el estadiaje histológico posquirúrgico de la muestra

\begin{tabular}{lc}
\hline Datos demográficos & \\
\hline Edad media & 63 años (40-85) \\
Hombres (75) & 65.5 años (42-85) \\
Mujeres (25) & 61.4 años (40-82) \\
& \\
Tipo de estudio RM e histología & 54 casos \\
Estadiaje primario & 50 \\
- Adenocarcinoma & 4 \\
- Sin malignidad & $14(5-46)$ \\
- N. ${ }^{\circ}$ ganglios (mediana) & 46 casos \\
Estadiaje posneoadyuvancia & 42 \\
- Adenocarcinoma & 4 \\
- Sin malignidad & $12(4-33)$ \\
- N. ${ }^{\circ}$ ganglios (mediana) & \\
Tipo de cirugía (exéresis del mesorrecto) & 80 casos \\
Abordaje anterior & 20 casos \\
Amputación abdominoperineal & \\
\hline
\end{tabular}

\begin{tabular}{|c|c|c|}
\hline \multicolumn{3}{|c|}{ Estadiaje histológico (pieza quirúrgica) } \\
\hline & Estudio primario & $\begin{array}{c}\text { Estudio } \\
\text { posneoadyuvancia }\end{array}$ \\
\hline \multicolumn{3}{|c|}{ Estadiaje local } \\
\hline 0 & 9 & 7 \\
\hline | & 15 & 12 \\
\hline$\| A$ & 10 & 9 \\
\hline IIB & 1 & 1 \\
\hline IIIA & 5 & 7 \\
\hline IIIB & 9 & 10 \\
\hline IIIC & 5 & 0 \\
\hline \multicolumn{3}{|c|}{ Tumor (T) } \\
\hline TO & 4 & 4 \\
\hline $\mathrm{T} 1-2$ & 25 & 24 \\
\hline T3 & 20 & 16 \\
\hline T4 & 5 & 2 \\
\hline \multicolumn{3}{|c|}{ Adenopatías positivas (N) } \\
\hline NO & 35 & 28 \\
\hline N1 & 10 & 15 \\
\hline N2 & 9 & 3 \\
\hline
\end{tabular}

sobreestadiaje e infraestadiaje, así como el acuerdo intragrupo entre múltiples observadores para las categorías dicotómicas definidas mediante el estadístico kappa de Fleiss; este se valoró según la escala propuesta por Landis y Koch (0.00-0.20: pobre; 0.21-0.40: ligera; 0.41-0.60: moderada; 0.61-0.80: considerable; 0.81-1.00: casi perfecta) ${ }^{25}$.

\section{Resultados}

La muestra incluyó 54 casos de estadificación primaria con cirugía posterior y 46 controles postQRT previos a la cirugía. Los datos demográficos y anatomopatológicos se presentan en la tabla 2. La tabla 3 muestra los resultados de precisión, sensibilidad, especificidad, VPP, VPN, RVP y RVN; todos los resultados expuestos corresponden a la estadificación locorregional final (incluyendo $T$ y $N$ ). Los valores fueron estadísticamente significativos $(p<0.05)$, salvo los correspondientes al AUC y la sensibilidad/especificidad postQRT sin DWI por los NER, así como la sensibilidad/ especificidad post-QRT por los RR tanto sin como con DWI. Al comparar las revisiones sin y con DWI, no se hallaron diferencias estadísticamente significativas.

Los ER mostraron una moderada disminución de la precisión con el uso de DWI tanto en el estadiaje primario como post-QRT (0.068 y 0.05 , respectivamente). En el resto de los parámetros hubo mínimos cambios con tendencia al empeoramiento al agregar DWI, destacando la caída de un $8.3 \%$ del VPN y un $6.7 \%$ la sensibilidad en el estadiaje primario, con un $3.7 \%$ de aumento del infraestadiaje (Tabla 4).

Por el contrario, los NER presentaron ligeras mejoras al añadir DWI, principalmente en el subgrupo post-QRT (aumento de 0.068 en precisión y $10.7 \%$ en especificidad; disminución de 0.12 en RVN y $4.5 \%$ en sobreestadiaje). Los RR mostraron peores resultados de AUC (0.037), especificidad (6.2\%) y VPN (5.3\%) en el estadiaje primario, sin otros cambios. Tampoco en el subgrupo post-QRT se produjeron grandes modificaciones, aunque destacó una mejora de la sensibilidad (6.4\%), el VPN (6.1\%) y la RVN (0.15), con disminución del infraestadiaje (3.8\%).

Las concordancias entre observadores del mismo grupo fueron de ligeras a moderadas (kappa: 0.23 a 0.6) en todos los grupos, sin identificar cambios destacables al añadir DWI, salvo un aumento en la concordancia post-QRT de los NER.

\section{Discusión}

En nuestro estudio no se han encontrado diferencias significativas en la distinción entre estadios iniciales y avanzados al añadir DWI a la HRT2w, en ninguno de los grupos de radiólogos ni en los subgrupos de pacientes. Sin embargo, destacó cierta tendencia a la obtención de peores resultados al añadir DWI en los ER, principalmente en el estadiaje primario.

Aunque existen numerosos estudios respecto a la valoración por RM del grado de infiltración por el tumor principal $(\mathrm{T})$ y la afectación ganglionar $(\mathrm{N})$, hay 
Tabla 3. Resultados del análisis estadístico

\begin{tabular}{|c|c|c|c|c|c|c|c|c|c|}
\hline & & AUC & Sensib. & Especif. & VPP & VPN & RVP & RVN & Kp \\
\hline \multicolumn{10}{|c|}{ Estadiaje global } \\
\hline \multirow[t]{2}{*}{ ER } & HRT2w & 0.735 & $87.1 \%$ & $50.4 \%$ & $69.9 \%$ & $74.7 \%$ & 1.75 & 0.25 & 0.49 \\
\hline & HRT2w + DWI & 0.675 & $83 \%$ & $48.1 \%$ & $67.9 \%$ & $68.1 \%$ & 1.6 & 0.35 & 0.54 \\
\hline \multirow[t]{2}{*}{ NER } & HRT2w & 0.653 & $82.7 \%$ & $40.5 \%$ & $63.5 \%$ & $65.2 \%$ & 1.39 & 0.42 & 0.55 \\
\hline & HRT2w + DWI & 0.693 & $84.2 \%$ & $45 \%$ & $65.7 \%$ & $69.4 \%$ & 1.53 & 0.35 & 0.48 \\
\hline \multirow[t]{2}{*}{$\mathrm{RR}$} & HRT2w & 0.637 & $79.8 \%$ & $37.2 \%$ & $62.7 \%$ & $58.1 \%$ & 1.27 & 0.54 & 0.44 \\
\hline & HRT2W + DWI & 0.623 & $82 \%$ & $32 \%$ & $61.5 \%$ & $57.2 \%$ & 1.2 & 0.56 & 0.48 \\
\hline \multicolumn{10}{|c|}{ Estadiaje primario } \\
\hline \multirow[t]{2}{*}{ ER } & HRT2w & 0.769 & $86.7 \%$ & $58.3 \%$ & $72.2 \%$ & $77.7 \%$ & 2.08 & 0.22 & 0.5 \\
\hline & HRT2w + DWI & 0.701 & $80 \%$ & $56.9 \%$ & $69.9 \%$ & $69.4 \%$ & 1.85 & 0.35 & 0.54 \\
\hline \multirow[t]{2}{*}{ NER } & HRT2w & 0.703 & $76.3 \%$ & $56.3 \%$ & $67.4 \%$ & $66.6 \%$ & 1.74 & 0.42 & 0.6 \\
\hline & HRT2w + DWI & 0.725 & $80.3 \%$ & $56.3 \%$ & $68.5 \%$ & $70.5 \%$ & 1.83 & 0.35 & 0.53 \\
\hline \multirow[t]{2}{*}{$\mathrm{RR}$} & HRT2w & 0.67 & $79.2 \%$ & $45.8 \%$ & $64.6 \%$ & $63.7 \%$ & 1.46 & 0.45 & 0.46 \\
\hline & HRT2w + DWI & 0.633 & $77.5 \%$ & $39.6 \%$ & $61.5 \%$ & $58.4 \%$ & 1.28 & 0.56 & 0.49 \\
\hline \multicolumn{10}{|c|}{ Estadiaje post-QRT } \\
\hline \multirow[t]{2}{*}{ ER } & HRT2w & 0.693 & $87.7 \%$ & $40.4 \%$ & $67.6 \%$ & $69.6 \%$ & 1.47 & 0.3 & 0.48 \\
\hline & HRT2w + DWI & 0.643 & $86.4 \%$ & $36.8 \%$ & $66 \%$ & $65.6 \%$ & 1.36 & 0.37 & 0.5 \\
\hline \multirow[t]{2}{*}{ NER } & HRT2w & $0.574^{*}$ & $90.5 \% *$ & $19.1 \% *$ & $60 \%$ & $60 \%$ & 1.11 & 0.49 & 0.23 \\
\hline & HRT2w + DWI & 0.642 & $88.9 \%$ & $29.8 \%$ & $62.9 \%$ & $66.6 \%$ & 1.26 & 0.37 & 0.34 \\
\hline \multirow[t]{2}{*}{$\mathrm{RR}$} & HRT2w & 0.594 & $80.6 \% *$ & $26.3 \% *$ & $60.8 \%$ & $48.7 \%$ & 1.09 & 0.73 & 0.4 \\
\hline & HRT2W + DWI & 0.608 & $87 \% *$ & $22.4 \% *$ & $61.4 \%$ & $54.8 \%$ & 1.12 & 0.58 & 0.44 \\
\hline
\end{tabular}

${ }^{*}$ Resultados estadísticamente no significativos.

Precisión diagnóstica (AUC, área bajo la curva), sensibilidad, especificidad, valores predictivos positivo y negativo (VPP y VPN), razones de verosimilitud positiva y negativa (RVP y RVN) y acuerdo intragrupo entre varios observadores (Kp) para la muestra completa de RM (Est. global) y las subcategorías de estadiaje primario o posneoadyuvancia (post-QRT). Los resultados se agrupan de acuerdo con el grado de experiencia de los radiólogos, así como según el uso únicamente de secuencias T2 de alta resolución (HRT2w) o con difusión añadida (HRT2w + DWI) para la evaluación.

ER: radiólogos con experiencia previa en estadiaje de cáncer de recto; NER: radiólogos sin experiencia previa en estadiaje; RR: residentes de radiología.

Tabla 4. Tasas de sobreestadiaje e infraestadiaje

\begin{tabular}{|c|c|c|c|c|c|c|c|}
\hline & & \multicolumn{2}{|c|}{ Est. global } & \multicolumn{2}{|c|}{ Est. primario } & \multicolumn{2}{|c|}{ Est. post-QRT } \\
\hline & & Sobreest. & Infraest. & Sobreest. & Infraest. & Sobreest. & Infraest. \\
\hline \multirow[t]{2}{*}{ ER } & HRT2w & $21.3 \%$ & $7.3 \%$ & $18.5 \%$ & $7.4 \%$ & $24.6 \%$ & $7.2 \%$ \\
\hline & HRT2w + DWI & $22.3 \%$ & $9.6 \%$ & $19.1 \%$ & $11.1 \%$ & $26 \%$ & $8 \%$ \\
\hline \multirow[t]{2}{*}{ NER } & HRT2W & $26.4 \%$ & $9.6 \%$ & $20 \%$ & $12.8 \%$ & $34.5 \%$ & $5.4 \%$ \\
\hline & HRT2W + DWI & $24.4 \%$ & $8.8 \%$ & $20 \%$ & $10.7 \%$ & $30 \%$ & $6.3 \%$ \\
\hline \multirow[t]{2}{*}{$\mathrm{RR}$} & HRT2w & $27 \%$ & $11.5 \%$ & $24 \%$ & $11.5 \%$ & $30.4 \%$ & $11.4 \%$ \\
\hline & HRT2W + DWI & $29.2 \%$ & $10.2 \%$ & $26.8 \%$ & $12.5 \%$ & $32 \%$ & $7.6 \%$ \\
\hline
\end{tabular}

Tasas de sobreestadiaje e infraestadiaje (\%) para la muestra completa de RM (Est. global) y las subcategorías de estadiaje primario o posneoadyuvancia (post-QRT). Los resultados se agrupan de la misma forma que en la tabla 3.

DWI: secuencias potenciadas en difusión; ER: radiólogos con experiencia previa en estadiaje de cáncer de recto; HRT2w: secuencias T2 de alta resolución; NER: radiólogos sin experiencia previa en estadiaje; RR: residentes de radiología

poca información sobre la eficacia diagnóstica del estadiaje locorregional. Sin embargo, este es clínicamente relevante para la toma de decisiones y aporta un acercamiento más representativo de la práctica diaria ${ }^{18}$. Únicamente hemos hallado tres artículos publicados al respecto y solo uno evalúa los cambios secundarios a añadir DWI, si bien con diferencias metodológicas que dificultan la comparación.

En un estudio sobre los cambios de la neoplasia secundarios a QRT visualizados solo con HRT2w, 

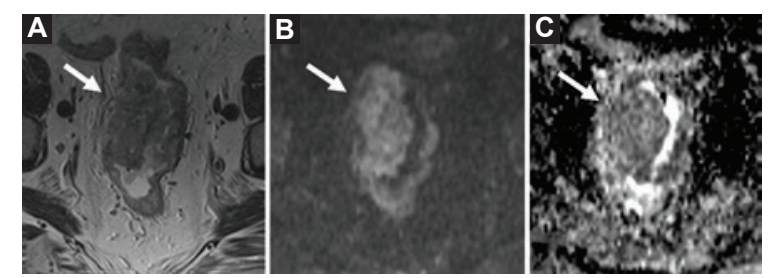

Figura 2. Ventaja de la difusión. Varón de 77 años con cáncer de recto alto, estadiaje primario. Secuencia axial TSE T2 de alta resolución. A: se observa una masa dependiente de la pared rectal (flecha) con prominencia hacia la luz; existen alteraciones de la grasa adyacente que en ocasiones podrían plantear dudas al diferenciar entre reacción desmoplásica e infiltración. B: la difusión muestra hiperintensidad de la masa con hipointensidad en ADC. C: compatibles con restricción, con pequeños tractos de similar comportamiento protruyendo hacia la grasa, lo que sugiere infiltración. En la pieza quirúrgica se confirmó un estadio IIA (T3NO).
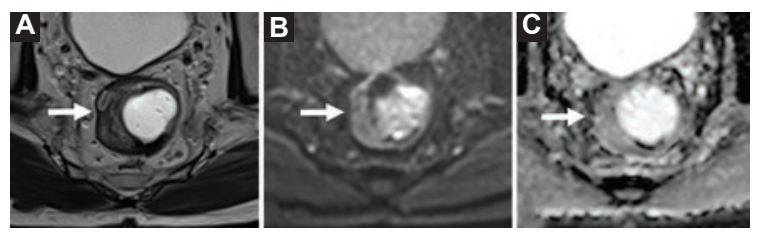

Figura 3. Limitación de la difusión. Varón de 68 años con cáncer de recto medio y quimiorradioterapia neoadyuvante. Control por RM previo a la cirugía. Secuencia axial TSE T2 de alta resolución. A: se aprecia un engrosamiento mural (flechas) con moderada heterogeneidad interna que puede plantear dudas sobre la persistencia de tumor viable, sin signos de infiltración adyacente. B: imagen en difusión y $A D C$. C: no se observan focos sospechosos de restricción evidente, lo que podría interpretarse como resolución completa. La pieza quirúrgica demostró un estadio I (yT1NO), con focos milimétricos aislados de tumor, no detectables con la técnica disponible.

Suppiah, et al. ${ }^{26}$ refieren una precisión para el estadio local del $43 \%$. Basándose en los datos brutos aportados en su artículo, para la determinación de estadios localmente avanzados (II-IV) pueden calcularse una sensibilidad del $82.8 \%$, una especificidad del $35.7 \%$, un VPP del $76.3 \%$ y un VPN del $45.4 \%$, próximos a los de nuestro estudio. Para la valoración postQRT con HRT2w y DWI, van den Broek, et al. ${ }^{27}$ hallaron una precisión global del $28-47 \%$ (incluyendo todos los estadios).

La mayor parte de los estudios sobre RM en el cáncer rectal se han realizado en centros de alto nivel con radiólogos muy experimentados. Aunque esta sería la circunstancia más deseable, ello no refleja necesariamente los resultados de la práctica diaria, en general en centros más pequeños y con profesionales menos especializados ${ }^{10,18}$. Sin embargo, es en estos casos en los que la DWI presentaría potencialmente mayor interés, pues estudios previos demostraron que puede facilitar la visualización y la delimitación del tumor (Fig. 2), así como la detección de ganglios perirrectales, por lo que podría ser de gran ayuda para los radiólogos en proceso de aprendizaje $e^{12,15,19,20}$.

En los grupos de radiólogos inexpertos de nuestro estudio, aunque la ausencia de significación limita las conclusiones, destaca cierta tendencia a una mejor valoración post-QRT al añadir DWI. Ello no es sorprendente, ya que las secuelas del tratamiento y los focos de persistencia tumoral pueden ser difíciles de valorar y diferenciar (Fig. 3) 6,28,29. Se ha descrito que la DWI puede ayudar a detectar y delimitar el tumor viable post-QRT ${ }^{20,30,31}$. Es razonable pensar que dicho beneficio de la DWI se haga más evidente con menos experiencia ${ }^{12,15,19,20}$, por lo que su uso podría ser recomendable al comienzo de la curva de aprendizaje. Únicamente Sassen, et al. ${ }^{32}$ hacen referencia a la correlación de los resultados con un radiólogo sin experiencia previa en el estadiaje del cáncer rectal, pero solo referida a la determinación de la respuesta completa post-QRT, por lo que no es comparable con nuestros resultados. Este mostró peor AUC (0.74 a $0.7)$ y VPP (100\% a $80 \%$ ) al agregar DWI, pero mejor sensibilidad (20 a $40 \%)$.

Nuestro estudio presenta varias limitaciones. En primer lugar, su carácter retrospectivo. Segundo, era posible cierto sesgo de aprendizaje, principalmente en los radiólogos menos experimentados, pero esto se evitó en la medida de lo posible al no aportar información sobre sus resultados a lo largo del estudio. Los sesgos de memoria se evitaron mediante aleatorización y separación entre lecturas. Tercero, los criterios de malignidad ganglionar en nuestro estudio siguen la recomendación de basarse solo en aspectos morfológicos ${ }^{8,9,33}$, sin aplicar las últimas recomendaciones de consenso publicadas coincidiendo con la fase final de adquisición de datos ${ }^{5}$. Sin embargo, en nuestra opinión, los resultados siguen siendo valiosos: a diferencia del tamaño (nuevo criterio añadido), los criterios morfológicos son subjetivos, por lo que es ahí donde la adición de DWI podría condicionar cambios más significativos $^{30,31}$.

\section{Conclusiones}

Los resultados del estudio no han demostrado la existencia de diferencias significativas en la distinción entre tumores rectales precoces y en estadios más avanzados al añadir DWI a HRT2w, independientemente de la experiencia previa de los radiólogos. 


\section{Financiamiento}

El presente trabajo ha sido financiado por la Fundación del Colegio de Médicos de Las Palmas de Gran Canaria (beca de investigación 2018). Dicho organismo no participó en el diseño del estudio, la recolección, el análisis y la interpretación de los datos, la redacción del artículo ni la decisión de enviarlo para su publicación.

\section{Conflicto de intereses}

Los autores declaran que no existe conflicto de intereses.

\section{Responsabilidades éticas}

Protección de personas y animales. Los autores declaran que para esta investigación no se han realizado experimentos en seres humanos ni en animales.

Confidencialidad de los datos. Los autores declaran que han seguido los protocolos de su centro de trabajo sobre la publicación de datos de pacientes.

Derecho a la privacidad y consentimiento informado. Los autores han obtenido el consentimiento informado de los pacientes y/o sujetos referidos en el artículo. Este documento obra en poder del autor de correspondencia.

\section{Bibliografía}

1. Visser BC, Varma MG, Welton ML. Local therapy for rectal cancer. Surg Oncol. 2001;10:61-9.

2. Demartines N, von Flüe MO, Harder FH. Transanal endoscopic microsurgical excision of rectal tumors: indications and results. World J Surg. 2001;25:870-5.

3. Glynne-Jones R, Wyrwicz L, Tiret E, Brown G, Rödel C, Cervantes A, et al. Rectal cancer: ESMO Clinical Practice Guidelines for diagnosis, treatment and follow-up. Ann Oncol. 2017;28:iv22-40.

4. Fowler KJ, Kaur H, Cash BD, Feig BW, Gage KL, Garcia EM, et al. ACR Appropriateness Criteria $₫$ Pretreatment Staging of Colorectal Cancer. J Am Coll Radiol. 2017;14:S234-44.

5. Beets-Tan RGH, Lambregts DMJ, Maas M, Bipat S, Barbaro B, Caseiro-Alves $F$, et al. Magnetic resonance imaging for clinical management of rectal cancer: updated recommendations from the 2016 European Society of Gastrointestinal and Abdominal Radiology (ESGAR) consensus meeting. Eur Radiol. 2017;23:1465-75.

6. Moreno CC, Sullivan PS, Kalb BT, Tipton RG, Hanley KZ, Kitajima HD, et al. Magnetic resonance imaging of rectal cancer: staging and restaging evaluation. Abdom Imaging. 2015;40:2613-29.

7. Prezzi D, Goh V. Rectal cancer magnetic resonance imaging: imaging beyond morphology. Clin Oncol. 2016;28:83-92.

8. Beets-Tan RGH, Lambregts DMJ, Maas M, Bipat S, Barbaro B Caseiro-Alves $\mathrm{F}$, et al. Magnetic resonance imaging for the clinical management of rectal cancer patients: recommendations from the 2012 European Society of Gastrointestinal and Abdominal Radiology (ESGAR) consensus meeting. Eur Radiol. 2013;23:2522-31.

9. Tudyka V, Blomqvist L, Beets-Tan RGH, Boelens PG, Valentini V, Van De Velde CJ, et al. EURECCA consensus conference highlights about colon \& rectal cancer multidisciplinary management: the radiology experts review. Eur J Surg Oncol. 2014;40:469-75

10. Lambregts DMJ, van Heeswijk MM, Delli Pizzi A, van Elderen SGC, Andrade L, Peters NHGM, et al. Diffusion-weighted MRI to assess response to chemoradiotherapy in rectal cancer: main interpretation pitfalls and their use for teaching. Eur Radiol. 2017;27:4445-54.

11. van der Paardt MP, Zagers MB, Beets-tan RGH, Stoker J, Bipat S. Patients who undergo preoperative chemoradiotherapy for locally advanced rectal cancer restaged by using diagnostic MR imaging : a systematic review and meta-analysis. Radiology. 2013;269:101-12.

12. Van Heeswijk MM, Lambregts DMJ, Palm WM, Hendriks BMF, Maas M, Beets GL, et al. DWI for assessment of rectal cancer nodes after chemoradiotherapy: is the absence of nodes at DWI proof of a negative nodal status? Am J Roentgenol. 2017;208:W79-84.

13. Lambregts DMJ, Vandecaveye V, Barbaro B, Bakers FCH, Lambrecht M, Maas M, et al. Diffusion-weighted MRI for selection of complete responders after chemoradiation for locally advanced rectal cancer: a multicenter study. Ann Surg Oncol. 2011;18:2224-31.

14. Feng $Q$, Yan $Y Q$, Zhu J, Xu JR. T staging of rectal cancer: accuracy of diffusion-weighted imaging compared with T2-weighted imaging on 3.0 tesla MRI. J Dig Dis. 2014;15:188-94.

15. Heijnen LA, Lambregts DMJ, Mondal D, Martens MH, Riedl RG, Beets GL, et al. Diffusion-weighted MR imaging in primary rectal cancer staging demonstrates but does not characterise lymph nodes. Eur Radiol. 2013;23:3354-60.

16. Zhou J, Zhan S, Zhu Q, Gong H, Wang Y, Fan D, et al. Prediction of nodal involvement in primary rectal carcinoma without invasion to pelvic structures: accuracy of preoperative CT, MR, and DWIBS assessments relative to histopathologic findings. PLoS One. 2014;9:1-8.

17. Zhang G, Cai YZ, Xu GH. Diagnostic accuracy of MRI for assessment of $T$ category and circumferential resection margin involvement in patients with rectal cancer: a meta-analysis. Dis Colon Rectum. 2016;59:789-99.

18. Blazic IM, Campbell NM, Gollub MJ. MRI for evaluation of treatment response in rectal cancer. Br J Radiol. 2016;89:20150964.

19. Marijnen CAM, Nagtegaal ID, Klein Kranenbarg E, Hermans J, van der Velde CJH, Leer JWH, et al. No downstaging after short-term preoperative radiotherapy in rectal cancer patients. J Clin Oncol. 2001:19:1976-84.

20. Lu ZH, Hu CH, Qian WX, Cao WH. Preoperative diffusion-weighted imaging value of rectal cancer: preoperative $T$ staging and correlations with histological T stage. Clin Imaging. 2016:40:563-8.

21. Hofheinz R-D, Wenz F, Post S, Matzdorff A, Laechelt S, Hartmann JT, et al. Chemoradiotherapy with capecitabine versus fluorouracil for locally advanced rectal cancer: a randomised, multicentre, non-inferiority, phase 3 trial. Lancet Oncol. 2012;13:579-88.

22. Brown G, Richards CJ, Bourne MW, Newcombe RG, Radcliffe AG, Dallimore NS, et al. Morphologic predictors of lymph node status in rectal cancer with use of high-spatial-resolution MR imaging with histopathologic comparison. Radiology. 2003;227:371-7.

23. Taylor FGM, Swift RI, Blomqvist L, Brown G. A systematic approach to the interpretation of preoperative staging MRI for rectal cancer. Am J Roentgenol. 2008:191:1827-35.

24. Colon and Rectum. En: Edge SB, Byrd DR, Compton CC, Fritz AG, Greene FL, Trotti A, editores. AJCC Cancer Staging Handbook. From AJCC Cancer Staging Man. $7^{\text {th }}$ ed. New York: Springer International Publishing; 2011. p. 143-59.

25. Landis JR, Koch GG. The measurement of observer agreement for categorical data. Biometrics. 1977;33:159-74.

26. Suppiah A, Hunter IA, Cowley J, Garimella V, Cast J, Hartley JE, et al. Magnetic resonance imaging accuracy in assessing tumour down-staging following chemoradiation in rectal cancer. Color Dis. 2009;11:249-53.

27. Van Den Broek JJ, Van Der Wolf FSW, Lahaye MJ, Heijnen LA, Meischl C, Heitbrink MA, et al. Accuracy of MRI in restaging locally advanced rectal cancer after preoperative chemoradiation. Dis Colon Rectum. 2017;60:274-83.

28. Vliegen RF, Beets GL, von Meyenfeldt MF, Kessels AGH, Lemaire EEMT, van Engelshoven JM, et al. Rectal cancer: MR imaging in local staging - is gadolinium-based contrast material helpful? Radiology. 2005;234:179-88.

29. Jhaveri KS, Hosseini-Nik H. MRI of rectal cancer: an overview and update on recent advances. Am J Roentgenol. 2015;205:W42-55.

30. Kim SH, Lee JM, Hong SH, Kim GH, Lee JY, Han JK, et al. Locally advanced rectal cancer: added value of diffusion-weighted MR imaging in the evaluation of tumor response to neoadjuvant chemo- and radiation therapy. Radiology. 2009;253:116-25.

31. Rao SX, Zeng MS, Chen CZ, Li RC, Zhang SJ, Xu JM, et al. The value of diffusion-weighted imaging in combination with T2-weighted imaging for rectal cancer detection. Eur J Radiol. 2008;65:299-303.

32. Sassen S, De Booij M, Sosef M, Berendsen R, Lammering G, Clarijs R, et al. Locally advanced rectal cancer: is diffusion weighted MRI helpful for the identification of complete responders (ypTON0) after neoadjuvant chemoradiation therapy? Eur Radiol. 2013;23:3440-9.

33. Moreno CC, Sullivan PS, Mittal PK. MRI evaluation of rectal cancer: staging and restaging. Curr Probl Diagn Radiol. 2017;46:234-41. 